\title{
Acceptance Tests of W7-X coils
}

H. Viebke (1), K. Hertel (1), J. Baldzuhn (1), G. Croari (1), L. Genini (2), B. Renard (2), L. Vieillard (2)

(1) Max-Planck-Institut für Plasmaphysik, Greifswald Branch, Euratom Association, Wendelsteinstraße 1, D-17491 Greifswald

(2) CEA Saclay, DSM/Irfu/SACM, F-91191 Gif sur Yvette Cedex

Corresponding author. Tel.: +49-3834-882742; Fax: +49-3834-882709;

E-mail address: holger.viebke@ipp.mpg.de

\begin{abstract}
ABSTRACT

WENDELSTEIN 7-X (W7-X) is a superconducting stellarator which uses 50 non-planar coils for the main confinement field and 20 planar coils for the magnetic configuration. All 70 coils are subject to an extensive test program after manufacturing and before assembly, realised at the cryomagnetic test facility at CEA Saclay. The tests are part of a well advanced quality assurance plan attesting the specified coil performance. The test program is mainly divided in warm and cold tests. Various tests have to be performed such as visual inspections, hydraulic measurements, electrical tests and the check of sensors. The most elaborate test is the cryogenic temperature margin test using current up to $17.6 \mathrm{kA}$ including the quench or fast discharge test and the evaluation of the specified interlayer joint resistance. Up to now $75 \%$ of the coils have been tested and accepted. The last acceptance tests at CEA are scheduled to be performed beginning of 2009. The test program has been enhanced permanently in its course, due to further improvement as well as considering aspects of the time schedule. This paper will present a short description of the test program done at CEA, their aim and background
\end{abstract}


Key words: WENDELSTEIN 7-X, superconducting coils, acceptance test,

\section{Introduction}

The plasma fusion experiment WENDELSTEIN 7-X (W7-X), which is under construction in the Max-Planck Institute for Plasma Physics (IPP) in Greifswald - Germany, consists of a modular system of 70 superconducting coils. 50 coils are for the generation of the main field having a non-planar shape and in addition 20 planar coils are for the field variation. The coils are wound from a cable in conduit conductor (CiCC). The coils are equipped with a case cooling loop and different instrumentation like temperature sensors, strain gauges and quench detection wires.

The detailed design and manufacture of the magnet components has been started in 1999. The production of the superconducting coils has been finished in 2008 and $75 \%$ of them has been already tested under cryogenic conditions. [1]. Each coil of W7-X is thoroughly tested before installation in a cryogenic test facility at the Commissariat a l'Energie Atomique (CEA) in Saclay, France. The tests before the final assembly are essential, because there is no access to the magnetic system of the final W7-X during operation. [2]

The first coil test for W7-X was done in 2003 using liquid helium and full current up to 17.6 kA. Since that time the number of coils tests per year has been increased significantly.

\section{Works acceptance test at manufacturers site}

A works acceptance of every coil will be performed under supervision of IPP before the cryogenic test will take place. The major tests are:

- Visual inspection and geometrical survey

- Insulation resistance using up to $13 \mathrm{kV}$-DC under various pressures 
- Inter turn resistance up to $2 \mathrm{kV}-\mathrm{AC}$ at atmospheric pressures

- Through flow test for the CiCC using helium gas

- Check of instrumentation (temperature, strain gauges, quench detection system)

After a successful made works acceptance test the coils will ship to the CEA cryogenic test facility for confirmation of the specified coil performance at cryogenic conditions.

\section{Incoming inspection and preparation for test}

The incoming inspection is the very first test proving the general status of the coil and to report damages caused by transport. After the visual inspection the coil will be mounted in a special frame supporting the coils during the time of testing. The following checks will be performed before the coil gets approval for installation into the cryostat:

- Temperature sensor check

- Strain gauge check

- Coil resistance and QD wire check

\section{Tests after installation}

Inside the cryostat is space for two coils. The two frames containing a coil are standing in top of each other (fig.1).The coils will connect to the cryostat electrically and hydraulically. A HV-DC-test up to $9.1 \mathrm{kV}$ and the check of QD-wires confirm the proper installation as well as leak test when the cryostat has been closed.

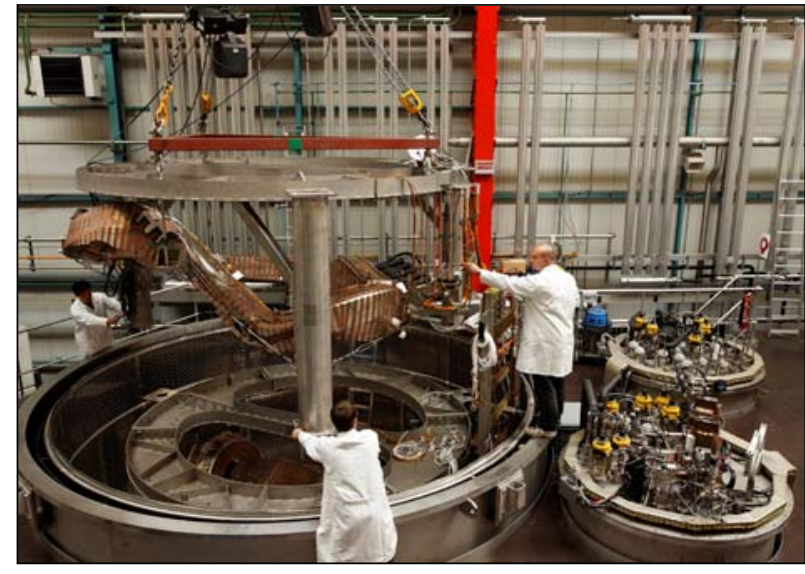

Fig.1 Mounting of a non-planar coil into the cryostat 


\section{Tests at cryogenic temperature}

When the cryostat is closed and the primary vacuum pump will start the evacuation. When the pressure is around $5^{*} 10^{-3}$ mbar the secondary vacuum system will take over the evacuation of the cryostat down to a pressure of $1 * 10^{-6}$ mbar. The evacuation of the cryostat takes around 24 hours. The cool down starts by the actively cooled shields of the cryostat filled with $\mathrm{LN}_{2}$. Helium circulates through the CiCC and the case cooling loops of the coils only [3] . In order to restrict the thermal stresses the cool down speed is limited to $2 \mathrm{~K} / \mathrm{h}$. The cool down takes around ten days. The measurement of the residual resistance ratio continues during the cool down by a 10A coil current. Two extensometers installed on the principal axis of the coil will monitor the shrinkage. At a temperature at $5.7 \mathrm{~K}$ the cool down has done and the current test can start. A first current test at $1 \mathrm{kA}$ including a fast discharge has to confirm the functionality of the quench detection system which is the main part of the safety concept during the current tests. The current test takes place at $17.6 \mathrm{kA}$ for the non-planar coils and $16 \mathrm{kA}$ for the planar coils. After one hour at full current a manual fast discharge interrupts the test. This causes a strong impact to the coil and will demonstrate if the coil withstands a similar scenario during future operation. The test facility has to be recovered due to the fast and significant increase of helium temperature and pressure before the second current can start. 


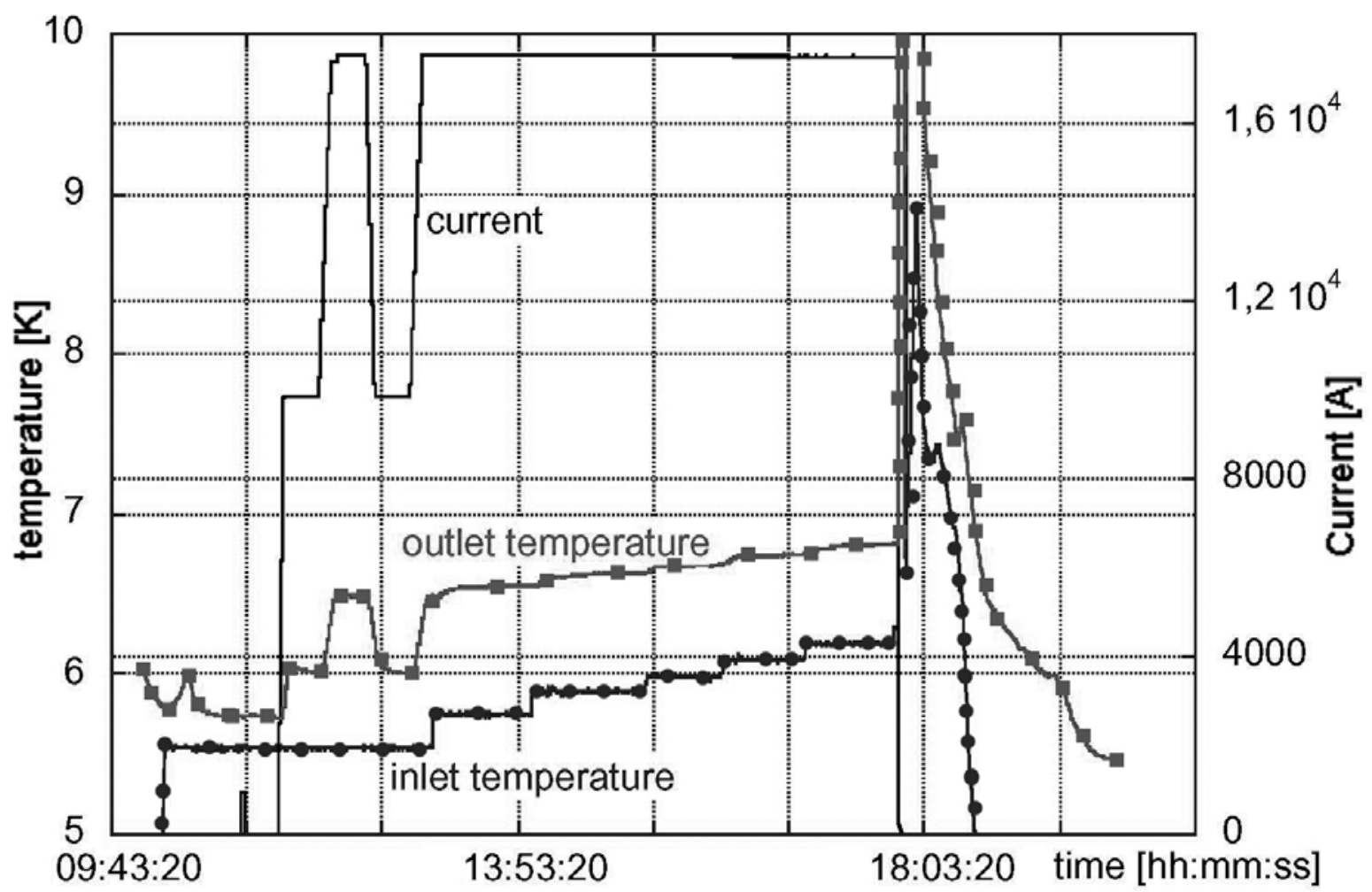

Fig.2 . Helium temperatures versus time during quench test

The first current test for one hour operates with an increased helium inlet temperature, which is $0.1 \mathrm{~K}$ below the quench temperature. The increased helium inlet temperature is $6.1 \mathrm{~K}$ for the non-planar coils and 7.3 $\mathrm{K}$ for the planar coils. Then the coil hast to be discharged by fast current discharge. After recovery the second current test has to verify the safety margin and has to be done at nominal current and at a test temperature which is $0.2 \mathrm{~K}$ below the critical temperature of the coil when operating in its self field. The critical temperature is given by calculations and common scaling laws taken from former quench tests (fig.2). The coil does not get quenching at the nominal current for one hour [4]. The resistance of the complete coil including the interlayer joints should be less than $6 \mathrm{nOhm}$ always.

The flow rate of the helium through the conductor is $0.6 \mathrm{~g} / \mathrm{s}$ and the cooling loop of $5 \mathrm{~g} / \mathrm{s}$ is tested at 6 bar nominal pressure. The pressure drops are monitored and limited to 1 bar for the conductor flow and 100 mbar for the case cooling. Another high voltage test using AC-and 
DC-voltage will confirm the specified insulation resistance of the coils at cryogenic conditions as well.

\section{Tests during warm up and before dispatch}

The warm up phase will be determined by the maximum tolerable temperature difference between the helium inlet and the casing of $40 \mathrm{~K}$. The warming up rate is limited to $2 \mathrm{~K} / \mathrm{h}$ above $100 \mathrm{~K}, 4 \mathrm{~K} / \mathrm{h}$ for $50 \mathrm{~K}<\mathrm{T}<100 \mathrm{~K}$ and $10 \mathrm{~K} / \mathrm{h}$ below $50 \mathrm{~K}$. The warm up of the coils is achieved mainly by breaking the vacuum and by using a power supply to warm up the coils. During warm up the following signals are monitored:

- Cryostat pressure

- Temperatures of the winding and the casing

- Strain and deformation of the casing

- Helium pressures in the winding and in the casing

\section{Data Acquisition and Documentation}

The data acquisition system records incessantly the following signals:

- Voltage drops

- Mass flow meters

- Current of Power supply

- Temperature sensors

- $\quad$ Pressure drops

- Strain gauges

- Extensometer

The sampling frequencies of the record depend on the period of test and the signal relevance. During cool down and warm up the sampling rate is $0.025 \mathrm{~Hz}$. The sampling rate is increased up to $5 \mathrm{kHz}$ for voltage drop and current in case of a quench test or a fast discharge. 
The test of each coil is documented in a final test report. Any deviation of the specified values or unexpected incidents during the test is reported in a Non Conformity Report (NCR).

\section{Frequent errors and improvements}

The operation of superconducting fusion magnet is a technical challenge and requires skilled engineers and pronounced quality awareness. Nevertheless a couple of errors appeared and had been solved. A number of removable connections inside the cryostat are required supplying the coils with current and helium. For example helium leaks and too high resistance of electrical contact plates required an interruption of the test run. The leak tests before start of cool down using local vacuum chambers are now well advanced and make the abort of test extremely rare. The contact plates have to be inspected regularly and are subject to a permanent refurbishing. The cleanliness of the helium circuit is another essential condition for an interference-free test cycle. The turbine of the cold helium circuit has been killed by frozen particles of oxygen or water sometimes. These accidents caused an expensive and time consuming repair. The time for regeneration of the helium circuit has been increased and the detection of pollution has been improved by using additional sensors. The content of water and oxygen is limited in a range of a single-digit parts per million.

\section{Conclusion}

The test program realised at the cryomagnetic test facility at CEA Saclay confirmed the well advanced design and proper quality of the magnets for the W7-X. All tested coils reached the specified key values. Experiences gained during the tests had influence to the further improvement of the coils. The cryogenic tests are a very helpful interface between manufacturing and final assembly as well as between W7-X and industry. It is a must to test large scale fusion magnets to full extent. 
References:

[1] F. Wagner et al., Physics, Technologies, and Status of the Wendelstein 7-X Device, 20thIAEA Fusion Energy Conf., Villamoura, 2004.

[2] Baldzuhn, J.;

Cold Test of the Superconducting Coils for the Stellarator for W7-X., MT-20 Conference on Magnet Technology, 27.08. - 31.08.2007, Philadelphia

[3] Bertrand Renard W7-X Superconducting Coils Cooling at the CEA Saclay Cryomagnetic Test Facility, SOFT conference, Rostock 2008

[4] Scope and Requirements for the Acceptance Tests on the W7-X - Coils 1-AAF-T0001.5 